\title{
Study of confined coherent acoustic phonon modes in a free-standing cubic GaN membrane by femtosecond spectroscopy
}

\author{
Chuan He, ${ }^{1, a)}$ Martin Grossmann, ${ }^{1}$ Delia Brick, ${ }^{1}$ Martin Schubert, ${ }^{1}$ Sergei V. Novikov, $^{2}$ \\ C. Thomas Foxon, ${ }^{2}$ Vitalyi Gusev, ${ }^{3}$ Anthony J. Kent, ${ }^{2}$ and Thomas Dekorsy ${ }^{1}$ \\ ${ }^{1}$ Department of Physics, University of Konstanz, 78457 Konstanz, Germany \\ ${ }^{2}$ School of Physics and Astronomy, University of Nottingham, Nottingham NG7 2RD, United Kingdom \\ ${ }^{3}$ Laboratoire d' Acoustique, LAUM, UMR CNRS 6613, LUNAM Université, Université du Maine, Le Mans, \\ France
}

(Received 3 July 2015; accepted 9 September 2015; published online 17 September 2015)

\begin{abstract}
Confined longitudinal coherent acoustic phonon modes are excited and detected in a sub- $\mu$ m-thick free-standing cubic GaN membrane by femtosecond pump-probe spectroscopy. After fs laser excitation, Brillouin oscillation and thickness oscillation with frequencies up to $100 \mathrm{GHz}$ are observed and studied in the time domain. We found an initial expansion of the membrane upon optical excitation at $400 \mathrm{~nm}$. Our experimental results confirmed earlier existing theoretical predictions and experimental observations that the hydrostatic deformation potential of cubic $\mathrm{GaN}$ is positive. (C) 2015 AIP Publishing LLC. [http://dx.doi.org/10.1063/1.4931495]
\end{abstract}

Time-resolved pump-probe spectroscopy has been applied to study ultrafast carrier dynamics and phononic properties of various semiconductors, such as $\mathrm{GaN},{ }^{1-4} \mathrm{GaAs},{ }^{5-7}$ and $\mathrm{Si}^{8-11}$ Among these, GaN is of great importance in optoelectronic and short-wavelength devices because of its widebandgap. $^{12-14} \mathrm{GaN}$ has two crystal structures: wurtzite (hexagonal) and zinc-blende (cubic). Hexagonal GaN, as the stable phase of $\mathrm{GaN}$ and the mostly employed phase for light emitting diodes, has attracted most of the research efforts. Coherent acoustic phonons (CAPs) in hexagonal GaN-based materials have been intensively investigated by optical pumpprobe spectroscopy. ${ }^{2,3,15-17}$ Recently, with the progresses in fabricating high-quality crystals, the metastable cubic-phase $\mathrm{GaN}$ (c-GaN) grown along the (001) direction has attracted much attention due to the absence of a spontaneous polarization and associated piezoelectric fields. ${ }^{18-20}$ Moss et al. reported about the first picosecond acoustic measurement in a $\mu$ m-thick c-GaN epilayer on a (001) GaAs substrate, where the CAP pulses are generated in an Al transducer. ${ }^{4}$ Using this method, the longitudinal sound velocity, elastic constants, and the refractive index of $\mathrm{c}-\mathrm{GaN}$ are obtained.

In this article, we prepared a sub- $\mu$ m-thick free-standing c-GaN membrane. A free-standing membrane was chosen due to its potential applications in optomechanics ${ }^{21,22}$ and its general interest for fundamental research. ${ }^{8-10,23}$ The fabrication process of the membrane followed the one in Refs. 24 and 25, and the details are described in the supplementary material. ${ }^{26}$ Confined longitudinal CAP modes in the $\mathrm{GHz}$ range are excited and detected in the membrane using femtosecond pump-probe spectroscopy based on asynchronous optical sampling (ASOPS). ${ }^{27,28}$ Instead of using a metallic transducer, we directly excite and detect the CAPs in c-GaN by two Titanium:sapphire lasers, where the central wavelength of pump and probe were both set to $800 \mathrm{~nm}$ and then frequency-doubled to $400 \mathrm{~nm}$ using BiBO crystals to reach the bandgap of c-GaN. The measurements were performed

a)chuan.he@uni-konstanz.de in reflection geometry, and the pump and probe beams were collinearly focused onto the membrane with a spot size below $2 \mu \mathrm{m}$ at nearly normal incidence. The pump fluence is $\sim 0.4 \mathrm{~mJ} / \mathrm{cm}^{2}$. After the absorption of the pump pulse, a large amount of non-equilibrium carriers is created. Acoustic phonons are created through the deformation potential (DP) and electron-phonon coupling, when the charge carriers transfer their excess energy to the lattice. The latter effect is described as thermoelastic (TE) effect. Since the main generation processes related to the photo-excitation of charge carriers are short compared to the phonon periods involved, a strain pulse consisting of longitudinal CAPs is generated and travels inside the membrane. The relative reflectivity change $\Delta \mathrm{R} / \mathrm{R}$ induced by the CAPs is detected by the time delayed probe pulses in a measurement window of $f_{\text {pump }}^{-1}=1.25 \mathrm{~ns} .{ }^{29}$ Because the optical quanta of the pump radiation, i.e., $h \nu=3.1 \mathrm{eV}$, is below the bandgap of $\mathrm{c}-\mathrm{GaN}\left(E_{g}=3.2 \mathrm{eV}\right.$ (Refs. 30 and 31)), the generation of CAPs in c-GaN is mainly through the DP.

Figure 1(a) shows the relative transient reflectivity change $\Delta R / R$ of the membrane. The observed drastic highamplitude transients in the reflectivity, which are taking place at the first hundred of picoseconds are presumably caused by the photo-excited charge carriers, while the acoustic contribution of much lower amplitude is clearly seen without any subtraction of the background contribution from the carriers (see inset in Fig. 1(a)). The acoustic contribution consists of a high-frequency Brillouin oscillation ${ }^{29}$ and a low-frequency triangle-wave-like oscillation. The Brillouin oscillation has a frequency of $(96.5 \pm 0.5) \mathrm{GHz}$ (Fig. 1(b)). According to $f_{\text {Brillouin }}=2 v_{\text {GaN }} n_{\text {GaN }} / \lambda_{0}$, where $v_{\text {GaN }}$ is the longitudinal sound velocity, $n_{\text {GaN }}$ the refractive index of $\mathrm{c}-\mathrm{GaN}$, and $\lambda_{0}$ the probe wavelength, we can extract the longitudinal sound velocity $v_{\text {GaN }}$ of c-GaN without knowledge of the membrane thickness $d_{\text {GaN. }}{ }^{29}$ Using $\lambda_{0}=400 \mathrm{~nm}$ and $n_{\text {GaN }}=2.66,{ }^{30} v_{\text {GaN }}$ is calculated to be $(7.26 \pm 0.05) \times 10^{3} \mathrm{~m} / \mathrm{s}$. The uncertainty in the estimated sound velocity is due to the finite width of the Brillouin spectral peak and the precision of the measurements of the 

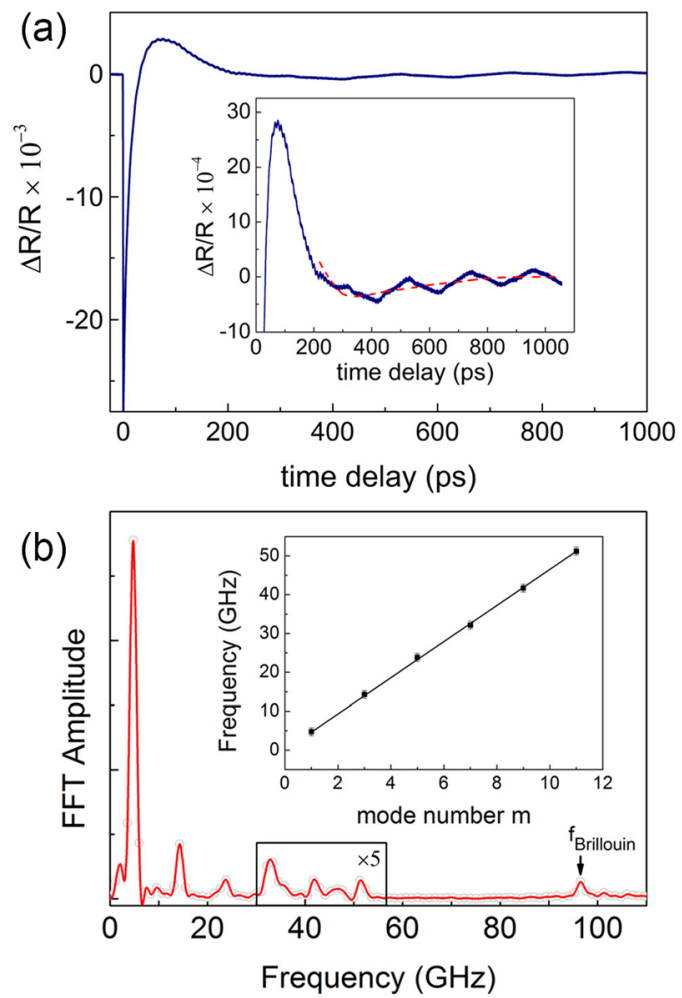

FIG. 1. (a) Transient reflectivity changes of the free-standing c-GaN membrane for pump and probe light both at $400 \mathrm{~nm}$. The inset is the close-up of the reflectivity signal, which is modulated by a high-frequency Brillouin oscillation and a low-frequency triangle-wave-like oscillation. The red dashed curve in the inset is the background. (b) Fast Fourier transform spectra of extracted triangle-wave-like oscillation from $\sim 200 \mathrm{ps}$. Due to the modulation of optical cavity thickness, the odd vibrational modes are observed. The inset is the plot of the frequency of the modes over the mode number. The peak at $96.5 \mathrm{GHz}$ is attributed to the Brillouin oscillation.

refractive index in Ref. 30. Starting from $\sim 200$ ps, the lowfrequency oscillation becomes noticeable and is almost undamped across the measurement window. The FFT result of triangle-wave-like oscillation presented in Fig. 1(b) shows a series of discrete acoustic phonon modes, which correspond to the odd vibrational modes of the membrane. ${ }^{8,32}$ The frequency of the modes follows: $f_{m}=m v_{G a N} / 2 d_{G a N}$, where the mode number $m=1,3, \ldots$, and 11 . The inset of Fig. 1(b) plots the frequency of the modes $f_{m}$ over the mode number $m$. Using $v_{\text {GaN }}$, the thickness of the membrane is determined to be $(780 \pm 7) \mathrm{nm}$, which is close to its nominal thickness. Furthermore, the longitudinal elastic constant of c-GaN is obtained: $C_{33}=\rho \cdot v_{\text {GaN }}^{2}=(316 \pm 2) \mathrm{GPa}$, where the density of c-GaN $\rho=5.99 \mathrm{~g} / \mathrm{cm}^{3}$ has been used. ${ }^{4}$ The Brillouin frequency in the membrane is slightly higher than that obtained from measurements on the supported c-GaN film $(94.2 \mathrm{GHz})$. This discrepancy might be related to the release of the interfacial strain after etching away the GaAs substrate, where the interfacial strain leads to the longitudinal elastic softening. ${ }^{33}$

Because of the large absorption depth in $\mathrm{c}-\mathrm{GaN}$ at $400 \mathrm{~nm},{ }^{30}$ the probe light partially reflects from both the front and back surfaces of the membrane, so the membrane can be considered as a Fabry-Perot etalon. For the vibrational modes in a free-standing membrane, only the odd modes cause the membrane thickness variation, which in turn modulates the reflectivity spectra. ${ }^{32}$ Here, we designate the center of the membrane at $z=0$, while the free surfaces of the membrane are at $z=z_{0}$ and $z=-z_{0}$, respectively. Consider the vibration of the membrane as a damped harmonics oscillator, the displacement $u_{z}^{m}(z, t)$ of the odd modes after optical excitation can be written as ${ }^{32}$

$$
u_{z}^{m}=A_{m} \sin \left(\frac{m \pi}{d_{\text {GaN }}} z\right) \cos \left(2 \pi f_{m} t+\phi\right) e^{-\frac{t}{\tau_{m}}}
$$

where $\tau_{m}$ the lifetime of the mode $m$. Thus, the thickness oscillations of the membrane caused by the odd acoustic displacement modes can by described by

$$
\Delta d=2 \sum_{m}(-1)^{\frac{m-1}{2}} A_{m} \cos \left(2 \pi f_{m} t+\phi\right) e^{-\frac{t}{\tau_{m}}}
$$

where $A_{m}$ is the amplitude of the vibrational mode $m$. $\Delta d$ corresponds to a symmetric vibration with respect to its middle plane $z=0$. At normal incidence, the stationary reflectivity $\mathbf{R}$ of the membrane is given by ${ }^{34}$

$$
\mathrm{R}=\frac{F \sin ^{2}\left(k_{0} D\right)}{1+F \sin ^{2}\left(k_{0} D\right)},
$$

where $F=4 r^{2} /\left(1-r^{2}\right)^{2}$ with $r=\left|\left(n_{\text {GaN }}-n_{\text {air }}\right) /\left(n_{\text {GaN }}+n_{\text {air }}\right)\right|$ is the reflection coefficient $\left(n_{\text {air }}\right.$ is the refractive index of air), $k_{0}=2 \pi / \lambda_{0}$, and $D\left(=n_{\text {GaN }} d_{\text {GaN }}\right)$ the optical thickness. The argument $k_{0} D$ can also be written as $\left(\pi f_{\text {Brillouin }}\right) /\left(2 f_{1}\right)$. At $\lambda_{0}=400 \mathrm{~nm}$, the reflectivity $\mathrm{R}$ for different thickness values is shown in Fig. 2, where the thickness variation of the membrane causes a concomitantly change in reflectivity

$$
\frac{\Delta \mathrm{R}}{\mathrm{R}}=\frac{2 k_{0}\left(n_{G a N}+\partial n_{G a N} / \partial \eta_{z z}\right)}{\tan \left(k_{0} D\right)\left[1+F \sin ^{2}\left(k_{0} D\right)\right]} \Delta d .
$$

Equation (2) indicates that the relative reflectivity change of the probe light is not only proportional to the variation of the membrane thickness $\Delta d$ but also proportional to the modulation of refractive index caused by the acoustic strain $\eta_{z z}=\Delta d / d_{G a N}$. These two modulation mechanisms are the so called optical cavity thickness (OCT) modulation and photoelastic (PE) modulation, respectively. ${ }^{8}$ Figure 3 shows the low frequency triangle-wave-like oscillation after subtracting the background. The theoretical result according to Eq. (2) is fitted to and compared with the experimental result in Fig. 3. A $1 / \mathrm{m}^{2}$ relationship for the amplitude of the odd modes, i.e., $\left|A_{m}\right|=|A| / m^{2}$, and a phase shift $\phi=\pi$ are needed for the fitting. Note that $A$ can be positive or negative for the cases where the photo-induced stresses cause the contraction, $\partial E_{g} / \partial P<0$, or expansion, $\partial E_{g} / \partial P>0$, of the membrane, respectively. In addition, the lifetime of the fundamental vibrational mode is extracted to be $\sim 1.7 \mathrm{~ns}$, despite of the short measurement window $(\sim 1.25 \mathrm{~ns})$. The fitting of the triangle-wave-like oscillation signal can be used to determine the sign of $A$ by comparing the phases of the trianglewave part of the experimental $\Delta R / R$ with the phase of the triangle-wave-like oscillations predicted by Eq. (2). Using the derived theoretical relations for fitting our experimental results, we found that in c-GaN the sign of the bandgap variation with pressure, $\partial E_{g} / \partial P$, is the same as that of the sum $\left(n_{\text {GaN }}+\partial n_{\text {GaN }} / \partial \eta_{z z}\right)$. This finding is in accordance with 


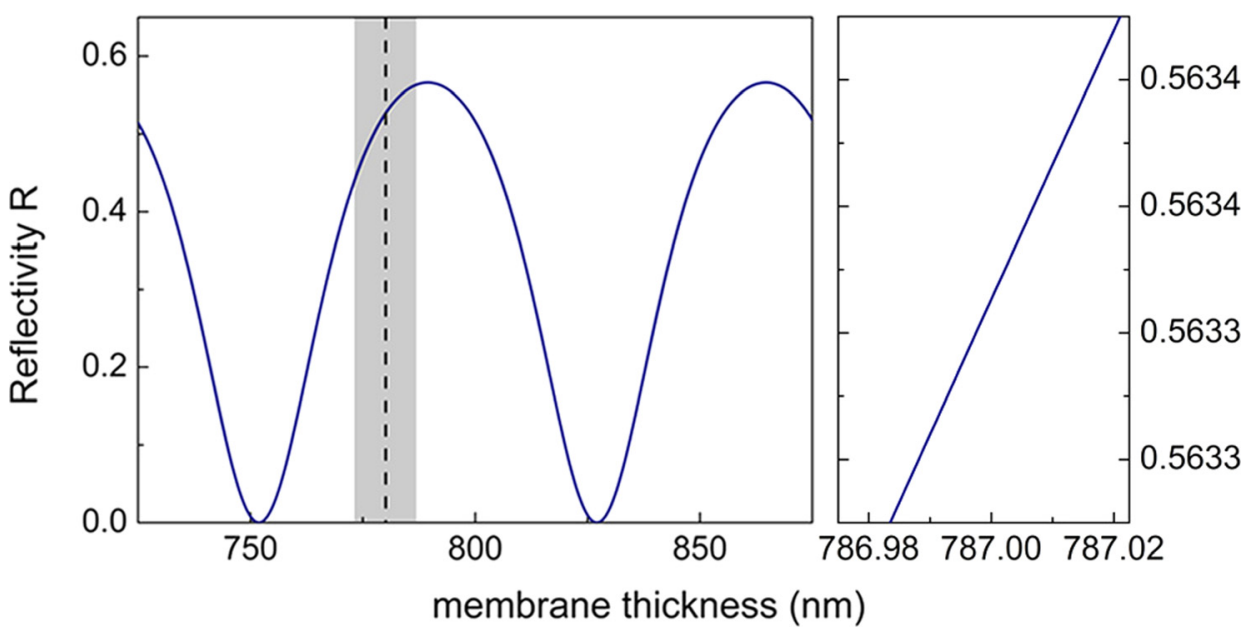

FIG. 2. (Left) Calculated stationary reflectivity of cubic GaN membrane at $400 \mathrm{~nm}$ wavelength depending on the membrane thickness. The gray area represents the error in the membrane thickness. (Right) Zoom into the thickness range around $787 \mathrm{~nm}$, where the variation in reflectivity is a linear function of the thickness variations.

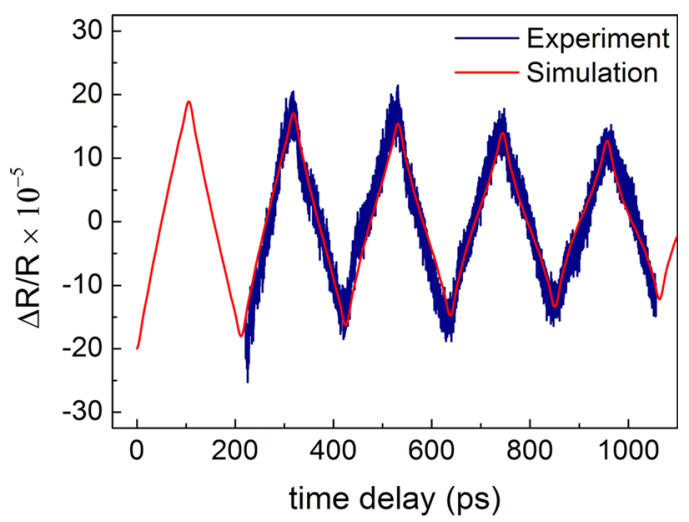

FIG. 3. The extracted triangle-wave-like oscillation (blue) and simulated results (red).

earlier existing theoretical predictions $\mathrm{s}^{31,35-38}$ and experimental observations ${ }^{39}$ that $\partial E_{g} / \partial P$ is positive and $\partial n / \partial P$ is negative, thus $\partial n / \partial \eta_{z z}$ is positive. It is worth mentioning that the theoretically predicted negative value of $\partial n / \partial P$ are obtained for the transparency region of c-GaN, ${ }^{31,36,37}$ hence can be applied to evaluate the sign of $\partial n / \partial P$ for our probe radiation, which is below the bandgap of c-GaN.

In conclusion, we prepared a free-standing c-GaN membrane and its coherent acoustic phonon dynamics is studied by femtosecond pump-probe spectroscopy in a measurement window of $\sim 1.25 \mathrm{~ns}$. The simultaneous observation of the Brillouin oscillation and the confined acoustic phonons modes in the membrane provides us a way to measure the thickness of the membrane with high accuracy. The ultrafast thickness oscillation is also observed and its contribution to the relative reflectivity change $\Delta R / R$ is analysed in the time domain. We obtain a positive value for the deformation potential of $\mathrm{c}-\mathrm{GaN}$.

This work was supported by the German Research Foundation (DFG) through the SFB 767 and the Engineering and Physical Sciences Research Council of the UK. The author gratefully acknowledges the financial support from the China Scholarship Council (CSC).

${ }^{1}$ C.-K. Sun, J.-C. Liang, X.-Y. Yu, S. Keller, U. K. Mishra, and S. P. DenBaars, Appl. Phys. Lett. 78, 2724 (2001).

${ }^{2}$ S. Wu, P. Geiser, J. Jun, J. Karpinski, J.-R. Park, and R. Sobolewski, Appl. Phys. Lett. 88, 041917 (2006).

${ }^{3}$ S. Wu, P. Geiser, J. Jun, J. Karpinski, and R. Sobolewski, Phys. Rev. B 76, 085210 (2007).

${ }^{4}$ D. Moss, A. V. Akimov, S. V. Novikov, R. P. Campion, C. R. Staddon, N. Zainal, C. T. Foxon, and A. J. Kent, J. Phys. D: Appl. Phys. 42, 115412 (2009).

${ }^{5}$ P. Babilotte, P. Ruello, D. Mounier, T. Pezeril, G. Vaudel, M. Edely, J. M. Breteau, V. Gusev, and K. Blary, Phys. Rev. B 81, 245207 (2010).

${ }^{6}$ O. B. Wright, B. Perrin, O. Matsuda, and V. E. Gusev, Phys. Rev. B 64, 081202 (2001).

${ }^{7}$ E. S. K. Young, A. V. Akimov, R. P. Campion, A. J. Kent, and V. Gusev, Phys. Rev. B 86, 155207 (2012).

${ }^{8}$ F. Hudert, A. Bruchhausen, D. Issenmann, O. Schecker, R. Waitz, A. Erbe, E. Scheer, T. Dekorsy, A. Mlayah, and J.-R. Huntzinger, Phys. Rev. B 79, 201307 (2009).

${ }^{9}$ J. Cuffe, O. Ristow, E. Chávez, A. Shchepetov, P. O. Chapuis, F. Alzina, M. Hettich, M. Prunnila, J. Ahopelto, T. Dekorsy, and C. M. S. Torres, Phys. Rev. Lett. 110, 095503 (2013).

${ }^{10}$ A. Bruchhausen, R. Gebs, F. Hudert, D. Issenmann, G. Klatt, A. Bartels, O. Schecker, R. Waitz, A. Erbe, E. Scheer, J. R. Huntzinger, A. Mlayah, and T. Dekorsy, Phys. Rev. Lett. 106, 077401 (2011).

${ }^{11}$ B. C. Daly, K. Kang, Y. Wang, and D. G. Cahill, Phys. Rev. B 80, 174112 (2009).

${ }^{12}$ D. Li, X. Sun, H. Song, Z. Li, Y. Chen, H. Jiang, and G. Miao, Adv. Mater. 24, 845 (2012).

${ }^{13}$ I. Akasaki, Proc. IEEE 101, 2200 (2013).

${ }^{14}$ A. Khan, K. Balakrishnan, and T. Katona, Nat. Photonics 2, 77 (2008).

${ }^{15}$ C.-K. Sun, J.-C. Liang, and X.-Y. Yu, Phys. Rev. Lett. 84, 179 (2000).

${ }^{16}$ R. Liu, G. D. Sanders, C. J. Stanton, C. S. Kim, J. S. Yahng, Y. D. Jho, K. J. Yee, E. Oh, and D. S. Kim, Phys. Rev. B 72, 195335 (2005).

${ }^{17}$ K.-H. Lin, C.-T. Yu, Y.-C. Wen, and C.-K. Sun, Appl. Phys. Lett. 86, 093110 (2005).

${ }^{18}$ S. V. Novikov, N. M. Stanton, R. P. Campion, C. T. Foxon, and A. J. Kent, J. Cryst. Growth 310, 3964 (2008).

${ }^{19}$ S. V. Novikov, C. T. Foxon, and A. J. Kent, Phys. Status Solidi A 207, 1277 (2010).

${ }^{20}$ S. Li, J. Schörmann, D. J. As, and K. Lischka, Appl. Phys. Lett. 90, 071903 (2007).

${ }^{21}$ D. J. Wilson, C. A. Regal, S. B. Papp, and H. J. Kimble, Phys. Rev. Lett. 103, 207204 (2009).

${ }^{22}$ J. D. Thompson, B. M. Zwickl, A. M. Jayich, F. Marquardt, S. M. Girvin, and J. G. Harris, Nature 452, 72 (2008).

${ }^{23}$ K. Usami, A. Naesby, T. Bagci, B. Melholt Nielsen, J. Liu, S. Stobbe, P. Lodahl, and E. S. Polzik, Nat. Phys. 8, 168 (2012).

${ }^{24}$ J.-H. Kim, D. H. Lim, and G. M. Yang, J. Vac. Sci. Technol., B 16, 558 (1998).

${ }^{25}$ J. Liu, K. Usami, A. Naesby, T. Bagci, E. S. Polzik, P. Lodahl, and S. Stobbe, Appl. Phys. Lett. 99, 243102 (2011).

${ }^{26}$ See supplementary material at http://dx.doi.org/10.1063/1.4931495 for the fabrication details of free-standing cubic GaN membrane.

${ }^{27}$ A. Bartels, R. Cerna, C. Kistner, A. Thoma, F. Hudert, C. Janke, and T. Dekorsy, Rev. Sci. Instrum. 78, 035107 (2007).

${ }^{28}$ R. Gebs, G. Klatt, C. Janke, T. Dekorsy, and A. Bartels, Opt. Express 18, 5974 (2010). 
${ }^{29}$ C. Thomsen, H. T. Grahn, H. J. Maris, and J. Tauc, Phys. Rev. B 34, 4129 (1986).

${ }^{30}$ U. Köhler, D. J. As, B. Schöttker, T. Frey, K. Lischka, J. Scheiner, S. Shokhovets, and R. Goldhahn, J. Appl. Phys. 85, 404 (1999).

${ }^{31}$ N. Bouarissa, Mater. Chem. Phys. 73, 51 (2002).

${ }^{32} \mathrm{R}$. Mindlin and J. Yang, An Introduction to the Mathematical Theory of Vibrations of Elastic Plates (World Scientific, 2006).

${ }^{33}$ B. M. Clemens and G. L. Eesley, Phys. Rev. Lett. 61, 2356 (1988).

${ }^{34}$ F. A. Jenkins and H. E. White, Fundamentals of Optics (McGraw-Hill, 1957).
${ }^{35}$ M. B. Kanoun, A. E. Merad, J. Cibert, H. Aourag, and G. Merad, J. Alloys Compd. 366, 86 (2004).

${ }^{36}$ Z. Usman, C. Cao, G. Nabi, D. Y. Kun, W. S. Khan, T. Mehmood, and S. Hussain, J. Phys. Chem. A 115, 6622 (2011).

${ }^{37}$ R. Riane, A. Zaoui, S. F. Matar, and A. Abdiche, Physica B: Condens. Matter 405, 985 (2010).

${ }^{38}$ M. B. Kanoun, S. Goumri-Said, A. E. Merad, G. Merad, J. Cibert, and H. Aourag, Semicond. Sci. Technol. 19, 1220 (2004).

${ }^{39}$ T. Lei, T. D. Moustakas, R. J. Graham, Y. He, and S. J. Berkowitz, J. Appl. Phys. 71, 4933 (1992). 